\title{
Reassessment of a meta-analysis of intraoperative cerebral oximetry-based management studies
}

\author{
Jason A. Davis, DPhil (1) • Rhodri Saunders, DPhil
}

Received: 4 October 2018/Revised: 13 October 2018/Accepted: 15 October 2018/Published online: 4 September 2019

(c) Canadian Anesthesiologists' Society 2019

\section{To the Editor,}

We read with interest the meta-analysis regarding intraoperative cerebral oximetry-based monitoring for maximizing perioperative outcomes by Zorrilla-Vaca et al. ${ }^{1}$ We note, however, some important discrepancies between the original source literature and the data that are used in the current analysis, which raise concerns. While conclusions regarding the primary outcome of cognitive impairment are not impacted, the means to reach that conclusion are at times not as accurate as they could be, and some of the secondary outcome conclusions differ in significance.

A primary example of discrepancy is in the studies used for the postoperative delirium outcome (Fig. 6). Of the six studies analyzed, three of them actually make no mention of "postoperative delirium", "POD", or "delirium" in the text or supplementary materials. ${ }^{2-4}$ Nor is there any mention of instruments typically used to assess POD in patients, such as the Confusion Assessment Method for the Intensive Care Unit (CAM-ICU). Of the remaining studies, the event counts used for Deschamps et al. appear to be for those receiving transfusions, not the delirium cases. ${ }^{5}$

A further example of data extraction concerns comes in the transfusion analysis in their Fig. 5. Values used for the study by Colak et al. (18 of 94 in the near-infrared

This letter is accompanied by a reply. Please see Can J Anesth 2019; 66: this issue.

Electronic supplementary material The online version of this article (https://doi.org/10.1007/s12630-019-01469-7) contains supplementary material, which is available to authorized users.

\section{J. A. Davis, DPhil $(\bowtie) \cdot$ R. Saunders, DPhil}

Coreva Scientific, Baden Württemberg, Germany

e-mail: jason@coreva-scientific.com spectroscopy (NIRS) group vs 24 of 96 in the control) are partly derived from values found in Fig. 1 of the source publication. ${ }^{6}$ Nevertheless, in Table 1 of the subsequent text, these values are noted to be percentages of patients (not absolute numbers of patients) who did not receive transfusions, meaning that the real numbers were 77 of 94 for NIRS and 73 of 96 for control, and thus change the direction of the effect.

The inclusion of the 2010 study by Cohn et al. ${ }^{7}$ represents a case where discrepancies in both data extraction and application of study inclusion criteria occur. The data extracted for length of hospital stay were described in the source paper as hospital-free days of the first 30 post-surgical days. Most importantly, Cohn et al. describe thenar placement of the oximetry probes, and we would therefore question inclusion of this study in a metaanalysis of cerebral NIRS-based management.

Following good practice, Zorrilla-Vaca et al. published their meta-analysis protocol (PROSPERO: CRD42017057293), but there were some deviations from the protocol that were not explained in their text. For example, the protocol describes continuous variable data synthesis using mean differences, which is changed to standardized mean differences in the final text. The latter is required when combining data on non-comparable scales, but the combined studies all used readily relatable units. For clinician readers, a mean change in hospital stay in days or hours is much more understandable than a proportional change in the standard deviation.

The protocol describes subgroup analyses by surgery and device, but the device analysis is not described. An analysis by intervention protocol was presented, but not mentioned in the protocol. This is important because the NIRS device provides data, but patient outcomes will only be influenced by the providers' response to those data. The 
authors classify seven of the studies as using a Denaulttype algorithm ${ }^{8}$ to correct cerebral desaturation. On closer examination, we suggest that nine of the studies generally follow the principal components of the algorithm. Regarding the subgroup results, in the text they indicate that for the primary outcome of Postoperative Cognitive Dysfunction (POCD), five of seven trials did not use the Denault algorithm yet yielded a significant effect of lower POCD. In fact, four studies did use the algorithm, and recalculation suggests the non-Denault algorithm studies trended towards non-significantly lower incidences of POCD. This difference is potentially crucial, as the suggestion that outcomes are improved regardless of how one responds to the NIRS data may not be supported.

Following our observations, we have re-analyzed the data from the studies indicated, except for the Cohn paper, which should not have been included. Details are found in the Electronic Supplementary Material eAppendix accompanying this submission, including the values used in the re-analysis and details of any assumptions or transformations of the source data undertaken. Subgroup analyses were performed for surgery class, device, and intervention algorithm.

As mentioned, most conclusions regarding outcomes do not change; NIRS-based monitoring is still significantly associated with a lower risk of postoperative cognitive impairment $(P=0.010)$ and with shorter ICU length of stay $(P<0.001)$. Subgroup analyses suggest, though, that other outcomes may be influenced by interactions among surgery type, device, or intervention algorithm. For example, POCD risk reduction may become insignificant if a Denault-type algorithm is not used (three studies; risk ratio, $0.82 ; 95 \%$ confidence interval, 0.60 to 1.13 ; $P=0.23$; intergroup $P=0.08$ ).

We emphasize that NIRS monitoring itself cannot change patient outcomes; instead, it will be the response of the clinical team to the NIRS data and application of clinical algorithms incorporating these data that may result in patient safety benefits. We agree with the caution expressed by Zorilla-Vaca et al. that results are subject to interpretation as it remains up to the reader to decide whether statistically significant results in such an analysis justify the modification of clinical practice; in any event, however, decisions should be based on the most transparent and accurate values possible.

Disclosures Coreva Scientific is a private consultancy that has received fees from Medtronic Inc. for analyses of medical devices. Medtronic played no role in initiating or influencing the current submitted work.

Conflicts of interest None declared.

Editorial responsibility This submission was handled by Dr. Hilary P. Grocott, Editor-in-Chief, Canadian Journal of Anesthesia.

\section{References}

1. Zorrilla-Vaca A, Healy R, Grant MC, et al. Intraoperative cerebral oximetry-based management for optimizing perioperative outcomes: a meta-analysis of randomized controlled trials. Can J Anesth 2018; 65: 529-42.

2. Murkin JM, Adams SJ, Novick RJ, et al. Monitoring brain oxygen saturation during coronary bypass surgery: a randomized, prospective study. Anesth Analg 2007; 104: 51-8.

3. Rogers CA, Stoica S, Ellis L, et al. Randomized trial of nearinfrared spectroscopy for personalized optimization of cerebral tissue oxygenation during cardiac surgery. Br J Anaesth 2017; 119: 384-93.

4. Vretzakis G, Georgopoulou S, Stamoulis K, et al. Monitoring of brain oxygen saturation (INVOS) in a protocol to direct blood transfusions during cardiac surgery: a prospective randomized clinical trial. J Cardiothorac Surg 2013; 8: 145.

5. Deschamps A, Hall $R$, Grocott $H$, et al. Cerebral oximetry monitoring to maintain normal cerebral oxygen saturation during high-risk cardiac surgery: a randomized controlled feasibility trial. Anesthesiology 2016; 124: 826-36.

6. Colak Z, Borojevic M, Bogovic A, Ivancan V, Biocina B, MajericKogler $V$. Influence of intraoperative cerebral oximetry monitoring on neurocognitive function after coronary artery bypass surgery: a randomized, prospective study. Eur J Cardiothorac Surg 2015; 47: 447-54.

7. Cohn SM, Pearl RG, Acosta SM, et al. A prospective randomized pilot study of near-infrared spectroscopy-directed restricted fluid therapy versus standard fluid therapy in patients undergoing elective colorectal surgery. Am Surg 2010; 76: 1384-92.

8. Denault A, Deschamps A, Murkin JM. A proposed algorithm for the intraoperative use of cerebral near-infrared spectroscopy. Semin Cardiothorac Vasc Anesth 2007; 11: 274-81.

Publisher's Note Springer Nature remains neutral with regard to jurisdictional claims in published maps and institutional affiliations. 